A. Fraj ${ }^{1}$, S. Arfa ${ }^{1}$, O. Jomaa ${ }^{1}$, F. Boubaker ${ }^{1}$, I. Bannour ${ }^{2}$, O. Berriche ${ }^{1}$, H. Sfar ${ }^{1}$ ${ }^{1}$ University Hospital Tahar Sfar Mahdia, Tunisia, Internal Medicine and Endocrinology Department, Mahdia, Tunisia; ${ }^{2}$ University Hospital Fattouma Bourguiba Monastir, TUNISIA, 2-Immunology Department, Monastir, Tunisia

Background: Multiple autoimmune syndrome (MAS) is a rare entity, defined by the association of three autoimmune diseases in the same patient. MAS can be classified into three groups.

Objectives: The objective of this work was to describe the autoimmune diseases profile in MAS in An Internal Medicine Department.

Methods: We report a retrospective analysis including 14 cases of MAS seen in The Internal Medicine Department at Taher Sfar Hospital, Mahdia, TUNISIA over a period of 10 years.

Results: We followed 14 patients with MAS. They were 14 women. The mean age of patients was 52 years. SAM was type 3 in 12 patients (85\%), type 2 in one patient $(7.1 \%)$ and one patient satisfied both type 2 and type 3 MAS criteria (7.1\%). No cases of MAS type 1 had been reported. We found 13 patients with 3 associated autoimmune diseases and one patient with 4 associated autoimmune diseases. The autoimmune diseases were: Sjögren's syndrome in 14 patients $(100 \%)$, Hashimoto's thyroiditis in 11 cases $(78 \%)$, systemic lupus erythematosus in 7 cases $(50 \%)$, Addison's disease in 4 cases $(28,5 \%)$, scleroderma, thymoma, vitiligo, Biermer and primary biliary cholangitis each in one case $(7.1 \%)$

Conclusion: Multiple autoimmune syndrome remains a rare but probably under-diagnosed entity. Thus, in patients with autoimmune disease, initial investigation and follow-up of clinical signs and biological stigmas of other autoimmune diseases should be cautious.

Disclosure of Interests: None declared

DOI: 10.1136/annrheumdis-2021-eular.3297

\section{AB0777 A RARE CASE OF A SOLITARY PLASMOCYTOMA OF A LUMBAR VERTEBRA}

D. Khalifa ${ }^{1}$, H. Hachfi ${ }^{1}$, N. Ben Chekaya ${ }^{1}$, M. Brahem ${ }^{1}$, M. Younes ${ }^{1} .{ }^{1}$ Taher Sfar Hospital, Rheumatology, Mahdia, Tunisia

Background: Solitary plasmocytoma is a rare tumour that represents around 2 to $5 \%$ of all plasma cell dyscrasias. It normally affects soft tissu but rarely the bone. Diagnosis is based on histology, the absence of bone marrow involvement Objectives: To drag attention to think of solitary plasmocytoma of bone when dealing with a vertebral fracture in the absence of the CRAB criteria of multiple myeloma. Methods: We report a rare case of a vertebral fracture of the 4th lumbar vertebra (L4) revealing a solitary plasmocytoma of bone.

Results: A 67-year-old female patient presented to our rheumatology department with back and left radicular pain of brutal onset, 15 days prior to her visit. Pain was severe and awakened her at nights. On examination, mobilities of the spine were unchanged but on palpation she had exquisite pain of L4. Laboratory tests showed a normal sedimentation rate of $15 \mathrm{~mm}$ the first hour, a negative c-reactive protein, normal calcemia and kidney tests. X-rays of lumbar spine showed a vertebral fracture with a destruction over $50 \%$ of the vertebral size and cortical rupture. MRI of the spine showed the absence of other lytic lesions or other fractures or spinal cord compression and showed the total destruction oft he anterior vertebral body of L4 (Figure 1). Protein electrophoresis was in normal range and 24h urinary proteinuria was negative. Other tests rules out gynecological, thyroid, and renal neoplasms. Sternal puncture showed a rich bone marrow of normal cells without further infiltration. Bone biopsy of the detected lesion showed tumour cells made of mature plasmocytes confirming the diagnosis of solitary plasmocytoma of the bone. The patient was treated with radiation therapy. The evolution after 24 months showed stabalised lesion and the absence of progression to multiple myeloma.

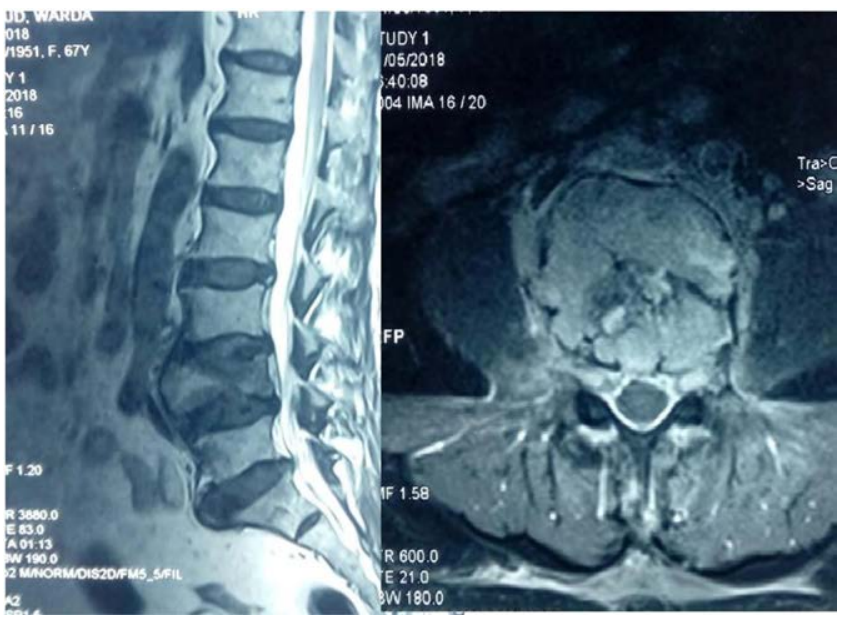

Figure 1. T2 weighted MRI showing the vertebral fracture of $L 4$
Conclusion: It is important to keep in mind the diagnosis of solitary plasmocytoma of bone when facing a solitary lesion or vertebral fracture despite relatively non agressive radiological signs. It is also important to note the possible evolution to multiple myeloma and keep a hawk-eyed guard.

\section{REFERENCES:}

[1] Masmoudi K, Elleuch E, Akrout R, et al. Le plasmocytome solitaire osseux: à propos de 3 cas et revue de la littérature. Pan Afr Med J; 25. Epub ahead of print 6 December 2016. DOI: 10.11604/pamj.2016.25.219.10933.

Disclosure of Interests: None declared

DOI: 10.1136/annrheumdis-2021-eular.3313

\section{AB0778 $\quad$ VENOUS THROMBOSIS IN UNUSUAL SITES}

A. Fraj ${ }^{1}$, O. Berriche ${ }^{1}$, S. Arfa ${ }^{1}$, O. Jomaa ${ }^{1}$, W. Romdhane ${ }^{1}$, H. Sfar ${ }^{1} .{ }^{1}$ University Hospital Tahar Sfar Mahdia, Tunisia, Internal Medicine and Endocrinology Department, Mahdia, Tunisia

Background: Unusual site venous thrombosis (USVT), which includes venous thromboses of the upper limbs, digestive tract, cellar and brain, are rarely observed compared to those of the lower limbs and require both an etiologica approach and a rigorous management.

Objectives: Our objective is to identify the epidemiological, clinical, therapeutic and evolutionary characteristics of USVT.

Methods: Retrospective study including 33 patients files with USVT followed in The Internal Medicine Departments at Tahar Sfar University Hospital Mahdia TUNISIA over a period of 10 years.

Results: They were 21 females $(63.6 \%)$ and 12 males $(36.4 \%)$ with an average age of $45.2 \pm 18$ years. Cerebral venous thrombosis was the most frequent localization identified in 10 patients (30.3\%), followed by gastrointestinal thrombosis in 9 cases $(27.3 \%)$, vena cava thrombosis in 9 cases $(27.3 \%)$ and upper limb thrombosis in 5 cases $(15.2 \%)$. The risk factors were respectively: age $(42.4 \%)$, obesity $(42.4 \%)$ smoking $(30 \%)$, prolonged downtime $(24.2 \%)$, surgical interventions $(6.1 \%)$ and pregnancy $(3 \%)$. An etiology was identified in 21 patients $(66.6 \%)$, dominated by constitutional thrombophilia (27.3\%), followed by BVL (15.1\%) and Behçet's disease $(16.4 \%)$. Rare causes were neoplasia in one case $(3 \%)$, scleroderma in one case (3\%) and inflammatory bowel disease in one case (3\%). The etiological investigation was negative in $36.3 \%$ of cases. Treatment was based on heparin therapy followed by vitamin $\mathrm{K}$ therapy. Progression was favourable without accident or recurrence in 25 patients $(75.5 \%)$. Complications were dominated by recurrence in $12.1 \%$ of cases, post-thrombotic disease in $6 \%$ of cases and pulmonary embolism in $3 \%$ of cases. We reported one death related to a hemorrhagic event.

Conclusion: USVT is a multifactorial pathology that involves both acquired and constitutional risk factors. Heparin therapy with an early relay by antivitamin $\mathrm{K}$ remains the treatment of choice today. The duration of treatment depend on the etiology, the recurrence of venous thrombosis and the risk of hemorrhagic complications.

Disclosure of Interests: None declared

DOI: 10.1136/annrheumdis-2021-eular.3332

\section{AB0779 CAN INFLAMMATION COEXIST IN PATIENTS WITH PROGRESSIVE PSEUDORHEUMATOID DYSPLASIA?}

$\underline{\text { S. Mahadevan }}{ }^{1}$, B. Navaneethan ${ }^{1}$, N. Gopalakrishnapillai Syamala ${ }^{1}$, M. Mamadapur ${ }^{1}$ R. S ${ }^{1}, \mathrm{~T} . \mathrm{Tn}^{1} .{ }^{1}$ Madras Medical College, Rheumatology, Chennai, India

Background: Progressive pseudorheumatoid dysplasia(PPRD) is considered as a degenerative genetic bone disorder. It is caused by loss of function mutation in WNT-1 inducible signaling pathway protein-3(WISP-3) ${ }^{1}$. WISP-3 gene function is required for the normal function of cartilage and skeletal development. The patients are normal at birth and start developing symptoms around 3-6 years of $\mathrm{age}^{2}$. The disease is characterised by stiffness, pain, deformity due to enlargement of the ends of short and long bones. Often, such patients are misdiagnosed as Juvenile idiopathic arthritis(JIA). In general, PPRD being considered as non-inflammatory disease, immunosuppressants or disease modifying anti rheumatic drugs(DMARDS) like methotrexate treatment are not used for treatment. Objectives: We report a patient with characteristic findings of PPRD but with coexisting clinical and imaging evidence of inflammation.

Methods: 16 year old male boy born of third degree consanguineous asymptomatic parents presented with progressive swelling, deformity of bilateral smal and large joints of upper and lower limbs. He also had pain in both hip and knee for past two years. Pain is associated with difficulty in walking and squatting. On examination he had bony enlargement around bilateral elbow, wrist, proximal and distal interphalangeal joints(Figure 1A). He also had restriction of bilatera hip movements and swelling of bilateral knee with effusion. He had exaggerated lumbar lordosis and flexion deformity of bilateral hip, knee. His blood counts, ESR, CRP were normal. Analysis of Knee joint synovial fluid showed cell count of 200/mm3 with no crystals and sterile culture. USG knee showed evidence of synovial thickening with increased power Doppler signals. Skeletal survey showed typical findings of PPRD with enlargement of epiphysis and osteoarthritis 
changes(Figure 1B). MRI hip showed minimal effusion, synovial thickening edema with STIR hyperintensity and enlargement of bilateral femoral epiphysis. MRI knee showed minimal effusion, marrow edema in patella(Figure 1C, arrow head), femoral condyle, diffuse synovial thickening with contrast enhancement(Figure 1D, arrow) and deformed patellar contour. Immunological tests showed negative RF, ACPA and positive ANA(Hep2) speckled 4+. Immunoblot for ENA was negative. His ophthalmological evaluation showed no evidence of uveitis.

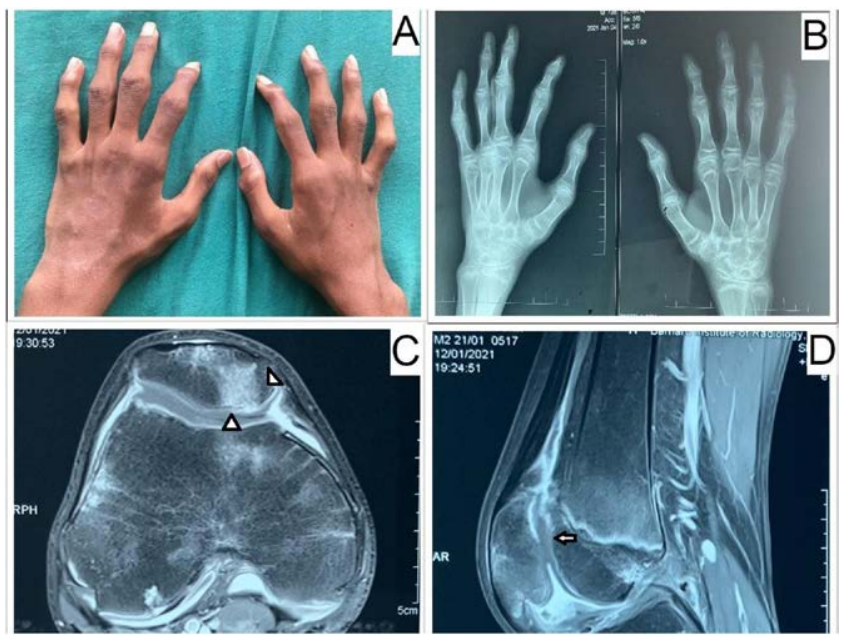

Figure 1. Clinical picture showing typical hand deformity and swelling at bone ends $(A)$, hand radiograph showing epiphyseal enlargement(B), MRI knee T2 STIR showing bone marrow edema(arrow head) in patella(C) and synovial thickening(arrow) with contrast enhancement in fat saturated T1 MRI with contrast.

Results: Patient tested positive for homozygous mutation in WISP-3 gene. He was treated with ibuprofen and supportive measures. Orthopedic consultation obtained and planned for hip, knee replacement during follow up. Follow up imaging and acute phase response was advised after three months.

Conclusion: Although PPRD was classically described as a degenerative disease, the findings presented in our case show coexisting inflammation. Bone marrow edema in weight bearing areas, synovial effusion may be explained as part of cartilage degeneration like in osteoarthritis but synovial hypertrophy with contrast enhancement, power Doppler signals in ultrasound, ANA positivity may be signs of coexisting inflammatory or autoimmune phenomenon.

REFERENCES:

[1] Hurvitz JR, Suwairi WM et al. Mutations in the CCN gene family member WISP3 cause progressive pseudorheumatoid dysplasia. Nat Genet. 1999 Sep;23(1):94-8. doi: 10.1038/12699. PMID: 10471507.

[2] Garcia Segarra N, Mittaz L et al. The diagnostic challenge of progressive pseudorheumatoid dysplasia (PPRD): a review of clinical features, radiographic features, and WISP3 mutations in 63 affected individuals. Am J Med Genet C Semin Med Genet. 2012 Aug 15;160C(3):217-29. doi: 10.1002/ ajmg.c.31333. Epub 2012 Jul 12. PMID: 22791401.

Disclosure of Interests: None declared

DOI: 10.1136/annrheumdis-2021-eular.3480

\section{AB0780 GENDER FEATURES OF VERIFICATION OF DIAGNOSIS UNDIFFERENTIATED ARTHRITIS}

O. Malyshenko ${ }^{1}$, M. Koroleva ${ }^{1}$, M. Letaeva ${ }^{1}$, J. Averkieva ${ }^{1}$, T. Raskina ${ }^{1}$ ${ }^{1}$ Kemerovo State Medical University, Department of Propedeutics of Internal Medicine, Kemerovo, Russian Federation

Background: Undifferentiated arthritis (UDA) is an inflammatory arthritis that does not meet the criteria for any rheumatologic disease. Early verification of UDA is currently one of the main goals of modern rheumatology, since a diagnosis established at an early date allows determining a therapeutic strategy. The high social significance of arthritis lies in the predominant lesion of people of working age, the steady progression of the disease, early disability and a reduction in life expectancy. Objectives: To study the gender characteristics of verification of the diagnosis of undifferentiated arthritis.

Methods: A retrospective analysis of 74 case histories of patients diagnosed with UDA was carried out. The study group consisted of 26 men and 48 women, mean age $50.6 \pm 4.3$ years. All patients underwent a comprehensive laboratory and instrumental examination according to the standard of an articular syndrome of unclear genesis.

Results: According to the data obtained, the duration of the articular syndrome averaged $2.53 \pm 1.2$ years. In 29 patients $(21.6 \%$ of women and $17.6 \%$ of men), on average, after $1.72 \pm 0.9$ years, the diagnosis of NDA was clarified. Taking into account modern diagnostic criteria, the following diseases were verified: rheumatoid arthritis in $13.5 \%$ (12.2\% in women and $1.3 \%$ in men), ankylosing spondylitis in $10.8 \%$ (2.7\% in women and $8.1 \%$ in men). Osteoarthritis, psoriatic arthritis and APS were diagnosed in $5.4 \%, 1.4 \%$ and $1.4 \%$ of women, and gouty arthritis bone tuberculosis and HIV in $4.1 \%, 1.4 \%$ and $1.4 \%$ of men respectively. In $60.8 \%$ (43.2\% in women and $17.6 \%$ in men), the etiology of arthritis was not verified. Conclusion: In a third of patients with UDA, diagnosis verification takes about 2 years on average. In more than half of patients, the diagnosis remains the same. According to the data obtained, rheumatoid arthritis was more often verified in women, while ankylosing spondylitis in men, which is consistent with statistical data. Disclosure of Interests: None declared

DOI: 10.1136/annrheumdis-2021-eular.3507

\section{ONE, NONE, MANY: REFLECTIONS ON A RARE CASE}

G. Sandri ${ }^{1}$, L. Belletti ${ }^{1}$, M. Cavedoni ${ }^{2}$, C. Galluzzo ${ }^{1}$, S. Bruni ${ }^{3}$, M. T. Mascia ${ }^{1}$ ${ }^{1}$ Rheumatology Institute, Department of Clinic and Medical Specialties, Modena, Italy; ${ }^{2}$ General Practitioner, Department of General Medicine, Modena, Italy; ${ }^{3}$ Genzyme, Orchard Therapeutics, Modena, Italy

Background: Rare diseases are all those diseases that present, in the European Union, a prevalence of less than 5 cases per 10,000 people. The number of rare diseases is estimated at roughly 7,000 but there are also longstanding medical conditions that elude diagnosis and could be identified as rare.

Objectives: Demonstrate the importance of international research in orphan diseases.

Methods: We report a case of $44 \mathrm{y} / \mathrm{o}$ female patient who arrived to our observation in 2006. Short stature, early puberty, ligament laxity, BMI <17. From the age of 29 : recurrent diarrhea, pain in the spine, osteolytic lesions in spine and endosteal thickening in long bones, muscle contractures, strength deficit, muscular hypotrophy and hypotonia, cardiac conduction and blood pressure disorders, demyelinating MS-lesions, hyperprolactinaemia, slow wound healing, sicca syndrome, osteoporosis. No familiarity for bone lesions. In 2007 her first son (21y/o) began to complain pain at limbs. The young man presented the same bone lesions as the mother and shortening of the PR, prolactinoma, recurrent diarrhea, short stature, early puberty. Over the years numerous pathologies have been first hypothesized and then excluded: multiple sclerosis, bone metastases, Paget's disease, celiac disease, McCune Albright, Camurati-Engelmann syndrome, mitochondrial disease. No conclusive diagnosis despite the thousands of kilometers traveled, the numerous experts heard and the countless examinations carried out by the patients.

Results: In September 2009, the patients had been investigated at the $\mathrm{NIH}$ (Washington D.C.) during the "Undiagnosed Diseases Program" but without results until 2013 when the patients were informed of the detection of an ATP6V1H gene mutation never described before in humans. The gene encodes a vacuolar ATPase, a multimeric enzyme that plays several roles: is involved in endocytosis, intracellular trafficking, and protein degradation and energy production, appears to be a risk factor in the development of dyslipidemias and type II diabetes, has a bone resorption function. Also in the patient's father were founded the same mutation and asymptomatic bone lesions. In 2016 and 2017 studies have reported mouse models of osteoporosis that were generated by knocking out the ATP6V1H gene.

Conclusion: from this case it is possible to understand the difficulty of diagnosing a rare disease, the need of an international collaboration in research From these studies it can be deduced moreover that the ATP6V1H gene could be an important target for therapeutic interventions aimed at preventing bone resorption and treating osteoporosis; evidence to support exploration of MMP9 and MMP13 as therapeutic targets for patients with ATP6V1H deficiency.This mutation seems to affect only one family, but it is possible that the penetrance of the disease-causing mutation is variable. In literature is reported an enhanced expression of MMP-9 in a variety of autoimmune diseases and neurologica pathologies (2) therefore the mutation can be at the basis of other much more common pathologies.

\section{REFERENCES:}

[1] Zhang $Y$, Huang $H$, Zhao G, Yokoyama T, Vega $H$, Huang $Y$, Sood R, Bishop K, Maduro V, Accardi J, Toro C, Boerkoel CF, Lyons K, Gahl WA, Duan X, Malicdan MC, Lin S. ATP6V1H Deficiency Impairs Bone Development through Activation of MMP9 and MMP13. PLoS Genet. 2017 Feb 3;13(2):e1006481. doi: 10.1371/journal.pgen.1006481.

[2] Ram M, Sherer Y, Shoenfeld Y. Matrix metalloproteinase-9 and autoimmune diseases. J Clin Immunol. 2006 Jul;26(4):299-307. doi: 10.1007/ s10875-006-9022-6.

Disclosure of Interests: Gilda Sandri: None declared, Lorenza Belletti: None declared, Michele Cavedoni: None declared, Claudio Galluzzo: None declared, stefano bruni Consultant of: Genzyme, Employee of: Genzyme, Maria Teresa Mascia: None declared

DOI: 10.1136/annrheumdis-2021-eular.3573 